\title{
Degeneracy of vector-channel spatial correlators in high temperature QCD
}

\author{
Christian Rohrhofer ${ }^{1, \star}$, Yasumichi Aoki ${ }^{2,3}$, Guido Cossu ${ }^{4}$, Hidenori Fukaya ${ }^{5}$, Leonid Glozman ${ }^{1}$, \\ Shoji Hashimoto ${ }^{2,6}$, Christian B. Lang ${ }^{1}$, and Sasa Prelovsek ${ }^{7,8,9}$ \\ ${ }^{1}$ Institute of Physics, University of Graz, 8010 Graz, Austria \\ ${ }^{2}$ KEK Theory Center, High Energy Accelerator Research Organization (KEK), Tsukuba 305-0801, Japan \\ ${ }^{3}$ RIKEN BNL Research Center, Brookhaven National Laboratory, Upton NY 11973, USA \\ ${ }^{4}$ School of Physics and Astronomy, The University of Edinburgh, Edinburgh EH9 3JZ, United Kingdom \\ ${ }^{5}$ Department of Physics, Osaka University, Toyonaka 560-0043, Japan \\ ${ }^{6}$ School of High Energy Accelerator Science, The Graduate University for Advanced Studies (Sokendai), \\ Tsukuba 305-0801, Japan \\ ${ }^{7}$ Faculty of Mathematics and Physics, University of Ljubljana, 1000 Ljubljana, Slovenia \\ ${ }^{8}$ Jozef Stefan Institute, 1000 Ljubljana, Slovenia \\ ${ }^{9}$ Institute für Theoretische Physik, Universität Regensburg, D-93040, Germany
}

\begin{abstract}
We study spatial isovector meson correlators in $N_{f}=2$ QCD with dynamical domain-wall fermions on $32^{3} \times 8$ lattices at temperatures up to $380 \mathrm{MeV}$ with various quark masses. We measure the correlators of spin-one isovector operators including vector, axial-vector, tensor and axial-tensor. At temperatures above $T_{c}$ we observe an approximate degeneracy of the correlators in these channels, which is unexpected because some of them are not related under $\mathrm{SU}(2)_{L} \times \mathrm{SU}(2)_{R}$ nor $\mathrm{U}(1)_{A}$ symmetries. The observed approximate degeneracy suggests emergent $\mathrm{SU}(2)_{C S}$ (chiral-spin) and $\mathrm{SU}(4)$ symmetries at high $T$.
\end{abstract}

\section{Introduction}

The behaviour of strongly interacting matter at high temperatures and its description in the phase diagram of QCD are important questions for a better understanding of the theory as well as for any kind of future applications. It is a priori not clear how two main features of QCD, confinement and chiral symmetry breaking, will behave in regions of higher temperature and/or density. For the latter it has been established that above a critial temperature QCD becomes chirally symmetric. In this regime the effects of the anomalous breaking of the $\mathrm{U}(1)_{A}$ symmetry are suppressed. There is strong evidence from lattice calculations using chirally-invariant fermions that the $\mathrm{U}(1)_{A}$ symmetry gets restored along with a gap opening in the eigenmode spectrum of the Dirac operator [1-3].

In this work we calculate spatial correlation functions of quark bilinears to probe their symmetry structure. The operators of interest are all possible local isovectors with spin $J=0$ and $J=1$. As fermion discretization we use the chirally invariant domain-wall formalism, and calculate the correlations at four temperatures from $\sim 1.2 T_{c}$ to $\sim 2.2 T_{c}$. The motivation for this is two-fold:

\footnotetext{
${ }^{\star}$ Speaker, e-mail: christian.rohrhofer@uni-graz.at
} 


\begin{tabular}{c|c|c|c|c|c|c}
\hline \hline$\beta$ & $m_{u d} a$ & $a[\mathrm{fm}]$ & \# configs & $L_{s}$ & $T[\mathrm{MeV}]$ & $T / T_{c}$ \\
\hline 4.10 & 0.001 & 0.113 & 800 & 24 & $\sim 220$ & $\sim 1.2$ \\
4.18 & 0.001 & 0.096 & 230 & 12 & $\sim 260$ & $\sim 1.5$ \\
4.30 & 0.001 & 0.075 & 260 & 12 & $\sim 320$ & $\sim 1.8$ \\
4.37 & 0.005 & 0.065 & 120 & 12 & $\sim 380$ & $\sim 2.2$ \\
\hline \hline
\end{tabular}

Table 1. Ensembles for $32^{3} \times 8$ lattices used in this work. $L_{s}$ is the length of the fifth dimension in the domain wall fermion formulation. The critical temperature for this set of parameters is $T_{c}=175 \pm 5 \mathrm{MeV}$. The mass range of degenerate up and down quarks $m_{u d}$ is $2-15 \mathrm{MeV}$.

- In the high temperature, chirally symmetric regime correlation functions connected by $\mathrm{SU}(2)_{L} \times$ $\mathrm{SU}(2)_{R}$ transformations are expected to become degenerate. The effects of $\mathrm{U}(1)_{A}$ breaking are expected to be suppressed. This regime eventually becomes $\mathrm{U}(2)_{L} \times \mathrm{U}(2)_{R}$ symmetric [4].

- In a series of numerical studies at $T=0$, where near-zero modes of the Dirac operator have been truncated from the quark propagators, extended $\mathrm{SU}(2)_{C S}$ (chiral-spin) and SU(4) symmetries [5, 6] have been observed in the hadron spectrum [7-10]. The low eigenmodes of the Dirac operator are strongly suppressed at high temperatures $[1,2]$, which raises the question whether these symmetries also occur naturally in the high $T$ spectrum.

\section{Lattices and operators}

The configurations are generated using the Symanzik action for the gauge sector and the Möbius domain wall formalism as fermion discretization [11, 12]. The length of the fifth dimension for the domain wall fermions is chosen separately for each ensemble in a manner which guarantees good chiral properties at moderate cost. The gauge links are stout smeared three times before the computation of the Dirac operator; the boundary conditions for quarks are set antiperiodic in $t$-direction and periodic in spatial directions. The ensembles and parameters including the lattice spacing $a$ are listed in Table 1, see also [2,13].

As observables we use correlators of local isovector operators $O_{\Gamma}(x)=\bar{q}(x) \Gamma \frac{\vec{\tau}}{2} q(x)$, where $\Gamma$ might be any element of the Clifford algebra; $\tau_{a}$ are the isospin Pauli matrices. We study spatial correlators in $z$-direction, as suggested in ref. [14]. Therefore we use a zero-momentum projection by summing over all lattice points in slices orthogonal to the measurement direction:

$$
C_{\Gamma}\left(n_{z}\right)=\sum_{n_{x}, n_{y}, n_{t}}\left\langle O_{\Gamma}\left(n_{x}, n_{y}, n_{z}, n_{t}\right) O_{\Gamma}(\mathbf{0}, 0)^{\dagger}\right\rangle
$$

Thus we can identify the propagating components of the Clifford algebra and organize them to spin $J=0$ and $J=1$ operators: The Pseudoscalar $(P S)$ is given by $\Gamma=\gamma_{5}$, and the Scalar $(S)$ operator by $\Gamma=\mathbb{1}$. For the Vector and Axial-vector operators $\Gamma$ has the following components:

$$
\mathbf{V}=\left(\begin{array}{l}
\gamma_{1}=V_{x} \\
\gamma_{2}=V_{y} \\
\gamma_{4}=V_{t}
\end{array}\right), \quad \mathbf{A}=\left(\begin{array}{l}
\gamma_{1} \gamma_{5}=A_{x} \\
\gamma_{2} \gamma_{5}=A_{y} \\
\gamma_{4} \gamma_{5}=A_{t}
\end{array}\right)
$$

Conservation of the vector current requires that $V_{z}$ does not propagate in $z$-direction. As the axial vector current $j_{5}^{\mu}$ is not conserved at zero temperature, the relevant component $\gamma_{3} \gamma_{5}$ of the Axialvector does propagate at zero temperature and eventually couples to the Pseudoscalar. Above the 


\begin{tabular}{|c|c|c|c|}
\hline Name & Dirac structure & Abbreviation & \\
\hline Pseudoscalar & $\gamma_{5}$ & $P S$ & \multirow{2}{*}{$\mathrm{U}(1)_{A}$} \\
\hline Scalar & $\mathbb{1}$ & $S$ & \\
\hline Axial-vector & $\gamma_{k} \gamma_{5}$ & $\mathbf{A}$ & \multirow{2}{*}{$\mathrm{SU}(2)_{A}$} \\
\hline Vector & $\gamma_{k}$ & $\mathbf{V}$ & \\
\hline Tensor-vector & $\gamma_{k} \gamma_{3}$ & $\mathbf{T}$ & \multirow{2}{*}{$\mathrm{U}(1)_{A}$} \\
\hline Axial-tensor-vector & $\gamma_{k} \gamma_{3} \gamma_{5}$ & $\mathbf{X}$ & \\
\hline
\end{tabular}

Table 2. Bilinear operators considered in this work and their transformation properties (last column). This classification assumes propagation in $z$-direction. The open vector index $k$ denotes the components $1,2,4$, i.e.

$$
x, y, t \text {. }
$$

critical temperature - after $\mathrm{U}(1)_{A}$ and $\mathrm{SU}(2)_{L} \times \mathrm{SU}(2)_{R}$ restoration $-A_{z}$ behaves as its parity partner $V_{z}$ and does not propagate in $z$-direction. For propagation in $z$-direction the tensor elements $\sigma_{\mu \nu}$ of the Clifford algebra are organized in the following way in components of Tensor- and Axial-tensor operators:

$$
\mathbf{T}=\left(\begin{array}{l}
\gamma_{1} \gamma_{3}=T_{x} \\
\gamma_{2} \gamma_{3}=T_{y} \\
\gamma_{4} \gamma_{3}=T_{t}
\end{array}\right), \quad \mathbf{X}=\left(\begin{array}{l}
\gamma_{1} \gamma_{3} \gamma_{5}=X_{x} \\
\gamma_{2} \gamma_{3} \gamma_{5}=X_{y} \\
\gamma_{4} \gamma_{3} \gamma_{5}=X_{t}
\end{array}\right)
$$

Table 2 summarizes our operators and gives the $\mathrm{U}(1)_{A}$ and $\mathrm{SU}(2)_{L} \times \mathrm{SU}(2)_{R}$ relations of these operators. Given restoration of the $\mathrm{U}(1)_{A}$ and $\mathrm{SU}(2)_{L} \times \mathrm{SU}(2)_{R}$ symmetries at high $T$ we expect degeneracies of correlators calculated with the corresponding operators.

For measurements at zero temperature the three components of the vectors give the same expectation value due to the $\mathrm{SO}(3)$ symmetry in continuum. On the finite temperature lattices the corresponding symmetry group is $D_{4 h}$ where the Vector has one two-dimensional $\left(V_{x}, V_{y}\right)$ and one onedimensional $\left(V_{t}\right)$ irreducible representation, and similar for $\mathbf{A}, \mathbf{T}, \mathbf{X}$. Thus only $x$ - and $t$-components are shown subsequently for the relevant operators.

\section{Results}

Figure 1 shows the spatial correlation functions normalized to 1 at $n_{z}=1$ for the operators given in Table 2. As argument we show $n_{z}$ which is proportional to the dimensionless product $z T$ for fixed $N_{t}$, the temporal extent of the lattice.

As we describe in more detail later, we find that all correlators connected by $\mathrm{SU}(2)_{L} \times \mathrm{SU}(2)_{R}$ and $\mathrm{U}(1)_{A}$ transformations coincide within small deviations at $T>220 \mathrm{MeV}$, which means that at these temperatures both chiral symmetries get restored. More interestingly, there are additional degeneracies of correlators. In total we observe three different multiplets:

$$
\begin{array}{ll}
E_{1}: & P S \leftrightarrow S \\
E_{2}: & V_{x} \leftrightarrow T_{t} \leftrightarrow X_{t} \leftrightarrow A_{x} \\
E_{3}: & V_{t} \leftrightarrow T_{x} \leftrightarrow X_{x} \leftrightarrow A_{t} .
\end{array}
$$

$E_{1}$ describes the Pseudoscalar-Scalar multiplet connected by the $\mathrm{U}(1)_{A}$ symmetry, that is realized in the absence of chiral zero-modes [15]. Note that we only consider the isospin triplet channels so $S$ corresponds to the $a_{0}$-rather than the $\sigma$-particle. The $E_{2}$ multiplet on the other hand contains some 


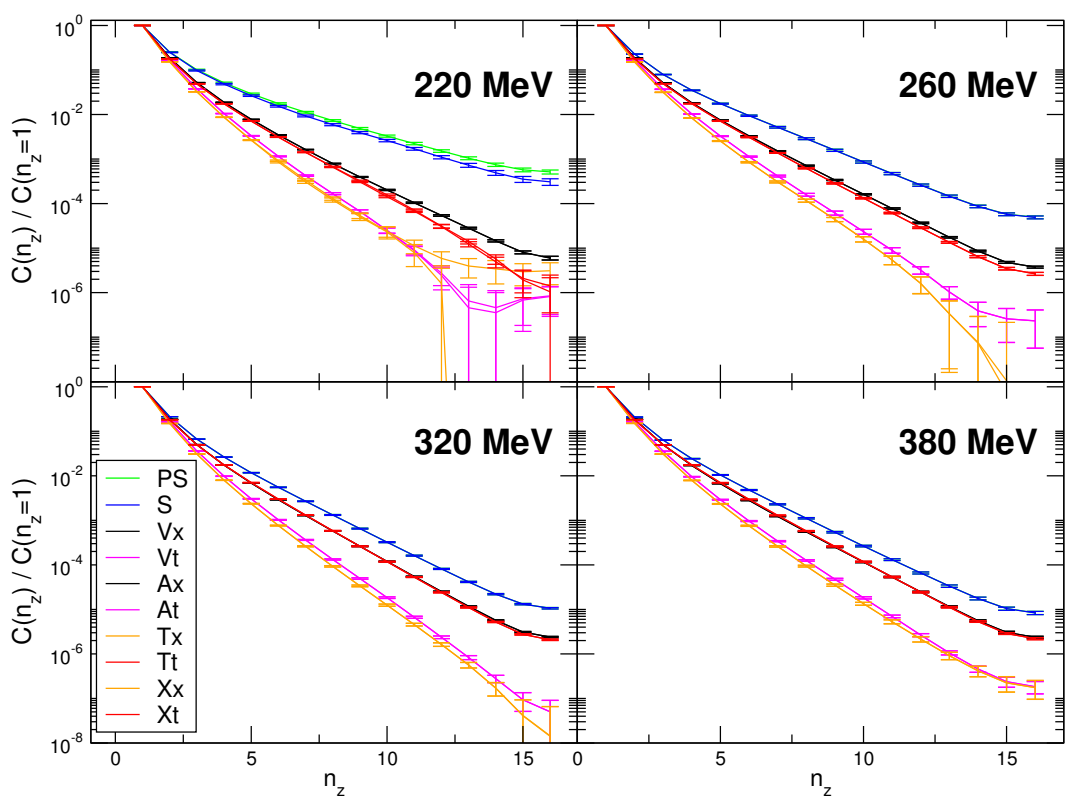

Figure 1. Normalized spatial correlators. The temperatures correspond to the ensembles listed in Table 1.

operators that are not connected by either $\mathrm{SU}(2)_{L} \times \mathrm{SU}(2)_{R}$ or $\mathrm{U}(1)_{A}$ transformations; this holds also for the $E_{3}$ multiplet.

The left side of Figure 2 shows the correlators of the $E_{1}$ and $E_{2}$ multiplets in detail at the highest available temperature $T=380 \mathrm{MeV}$. Here we also show correlators calculated with non-interacting quarks. The non-interacting (free) data have been generated on the same lattice sizes using a unit gauge configuration and verified by analytic calculation. Due to the small quark mass the differences between chiral partners is negligible for the free case, therefore they are omitted.

We observe a precise degeneracy between $S$ and $P S$ correlators, which is consistent with the $\mathrm{U}(1)_{A}$ restoration on these lattice ensembles [1]. The logarithmic slope of the interacting (dressed) $S$ and $P S$ correlators is substantially smaller than that for free quarks. In the latter case the slope is given by $2 \pi / N_{t}$. A system of two free quarks cannot have 'energy' smaller than twice the lowest Matsubara frequency [14]. For the $E_{2}$ multiplet we observe asymptotic slopes that are quite close to $2 \pi / N_{t}$ in agreement with previous studies [16].

Figures 3 and 4 show ratios of correlators connected by various symmetries at different temperatures. We use these ratios as quantities to measure the level of symmetry breaking. Figure 3 shows ratios of $P S$ and $S$ correlators on the left, and ratios of $V_{x}$ and $A_{x}$ correlators on the right. Figure 4 shows ratios of $X_{t}$ and $T_{t}$ correlators on the left, as well as of $V_{x}$ and $T_{t}$ correlators on the right side. The chiral symmetries $\mathrm{U}(1)_{A}$ and $\mathrm{SU}(2)_{L} \times \mathrm{SU}(2)_{R}$ are restored at $T>220 \mathrm{MeV}$, as is evident from both Figures (see also, e.g. $[17,18]$ ). Interesting is the level of residual breaking at $T=220 \mathrm{MeV}$ : The corresponding deviation from unity for $U(1)_{A}$ connected correlators is at least one order of magnitude higher than for $\mathrm{SU}(2)_{L} \times \mathrm{SU}(2)_{R}$ connected correlators. ${ }^{1}$

\footnotetext{
${ }^{1}$ This effect might be spoiled by chirality violating eigenmodes of the Dirac operator, which are non-neglible for this ensemble and temperature [2]. They are absent for higher temperatures.
} 

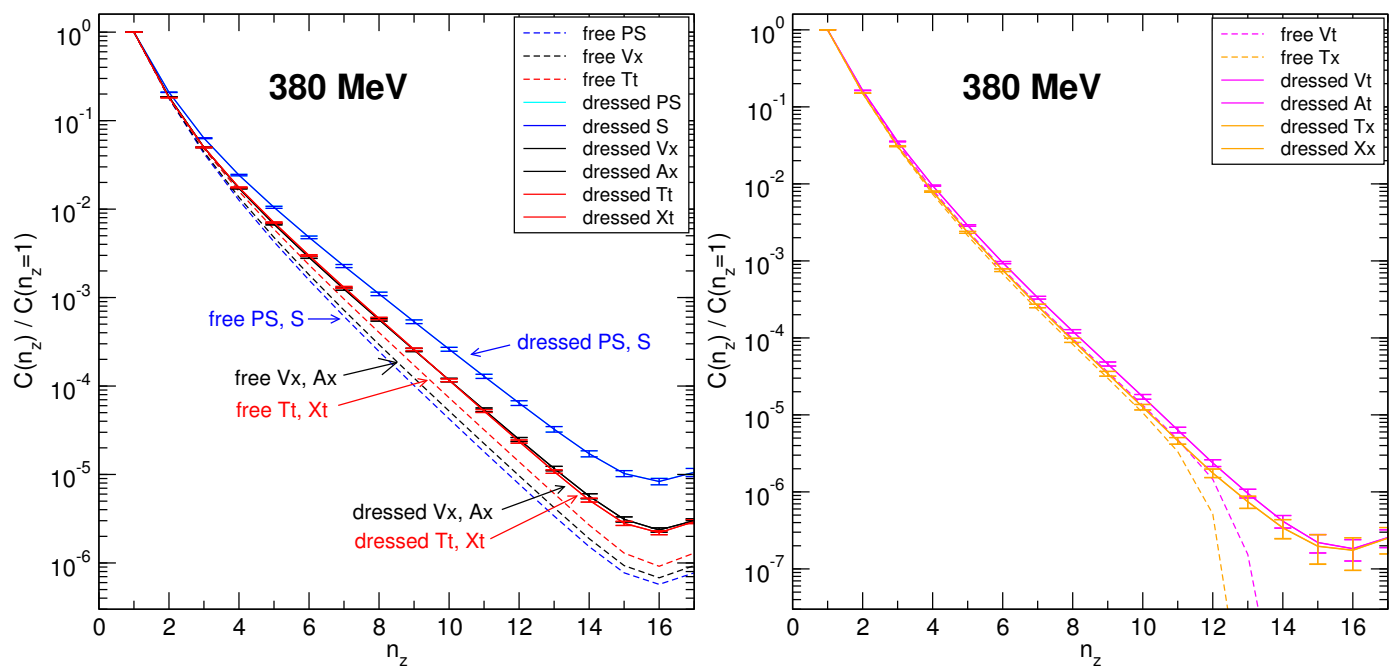

Figure 2. Left side: $E_{1}$ and $E_{2}$ multiplets (4-5) for interacting (dressed) and non-interacting (free) calculations at $T=380 \mathrm{MeV}$. Right side: $E_{3}$ multiplet (6) for interacting (dressed) and non-interacting (free) calculations.
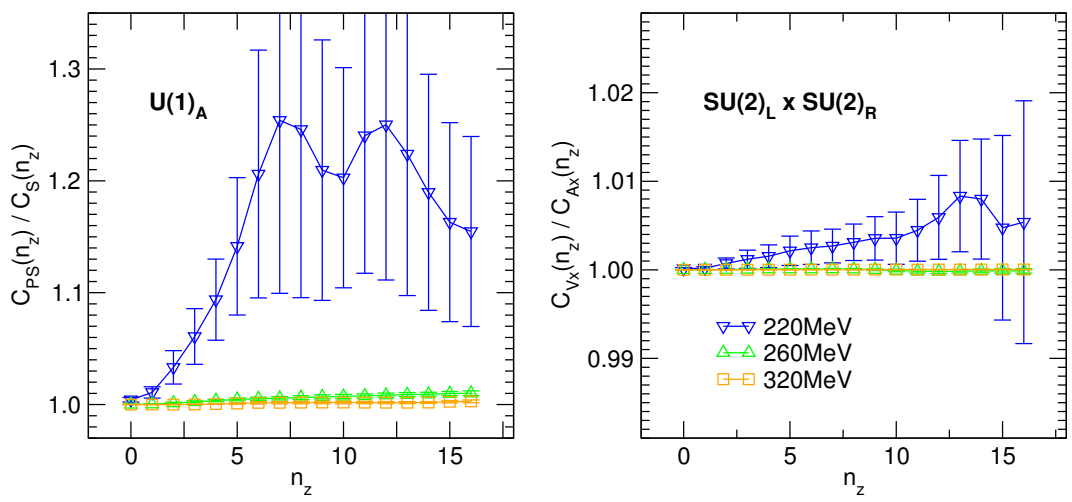

Figure 3. Ratios of $P S$ and $S$ as well as of $V_{x}$ and $A_{x}$ correlators from Figure 1, that are related by chiral $\mathrm{U}(1)_{A}$ and $\mathrm{SU}(2)_{L} \times \mathrm{SU}(2)_{R}$ transformations.

Figures 2 and 4 suggest a possible higher symmetry that connects $V_{x}$ and $T_{t}$ channels. The right panel of Figure 4 shows the corresponding ratio, which demonstrates an approximate degeneracy at the level of $5 \%$ above $T \simeq 320 \mathrm{MeV}$. We notice that this degeneracy is not expected in the free quark limit which is plotted by a dashed curve. This unexpected symmetry requires that the cross-correlator calculated with the $V_{x}$ and $T_{t}$ operators (both create the $1^{--}$states) should vanish. We have carefully checked that it indeed vanishes to high accuracy.

The right side of Figure 2 shows the $E_{3}$ multiplet. Here again we observe a precise degeneracy in all $\mathrm{SU}(2)_{L} \times \mathrm{SU}(2)_{R}$ and $\mathrm{U}(1)_{A}$ connected correlators, as well as the approximate degeneracy in all four correlators. We also see qualitatively different data between free and dressed correlators at $n_{z} \geq 11$, as also seen in [19]. For this set of operators the spatial correlations of free quarks become 

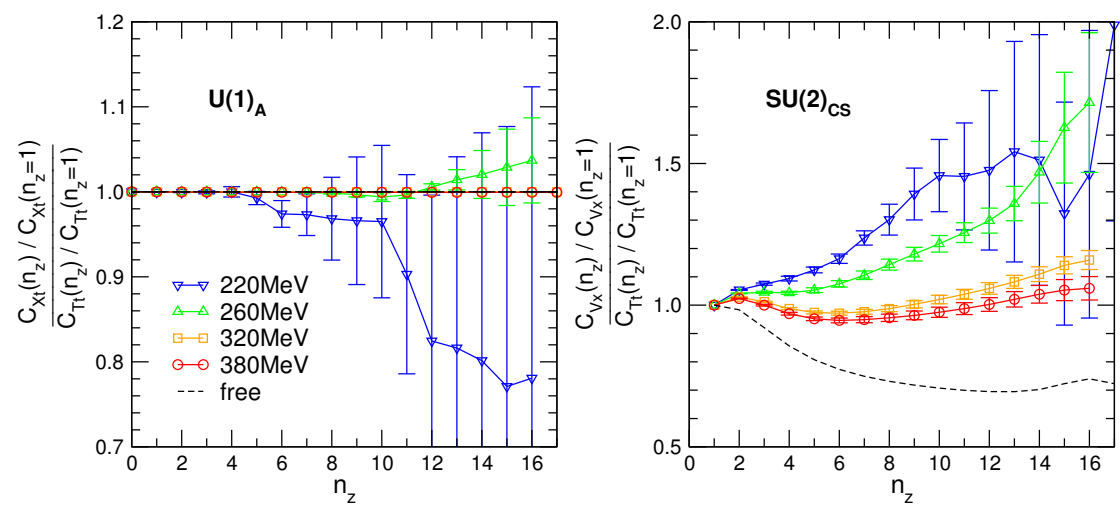

Figure 4. Ratios of normalized $X_{t}$ and $T_{t}$ as well as of $V_{x}$ and $T_{t}$ correlators from Figure 1, that are related by $\mathrm{U}(1)_{A}$ and $\mathrm{SU}(2)_{C S}$ transformations.

negative near the symmetry point, which is a finite size effect. The absence of this behaviour in the dressed correlators implies that we do not observe free non-interacting quarks but instead systems with some interquark correlation, which is in accordance with the known results for energy density and pressure at high temperatures [20].

\section{$4 \mathrm{SU}(2)_{C S}$ and $\mathrm{SU}(4)$ symmetries}

In this section we introduce the $\mathrm{SU}(2)_{C S}$ and $\mathrm{SU}(4)$ transformations, which connect operators from multiplet $E_{2}(5)$ as well as from multiplet $E_{3}(6)$ and contain chiral transformations as a subgroup. The basic ideas of $\mathrm{SU}(2)_{C S}$ and $\mathrm{SU}(4)$ symmetries at zero temperature are given in [6]. Here we adapt the group structure to our setup.

We use the $\gamma$-matrices given by

$$
\begin{aligned}
\gamma_{i} \gamma_{j}+\gamma_{j} \gamma_{i} & =2 \delta_{i j} \\
\gamma_{5} & =\gamma_{1} \gamma_{2} \gamma_{3} \gamma_{4} .
\end{aligned}
$$

The generators of the $\mathrm{SU}(2)_{C S}$ chiral-spin group, defined in the Dirac spinor space and diagonal in flavour space, are given by

$$
\vec{\Sigma}=\left\{\gamma_{k},-i \gamma_{5} \gamma_{k}, \gamma_{5}\right\}
$$

$\mathrm{SU}(2)_{C S}$ contains $\mathrm{U}(1)_{A}$ as a subgroup. The $\mathfrak{s u}(2)$ algebra $\left[\Sigma_{\alpha}, \Sigma_{\beta}\right]=2 i \epsilon^{\alpha \beta \gamma} \Sigma_{\gamma}$ is satisfied with any $k=1,2,3,4$. The $\mathrm{SU}(2)_{C S}$ transformations mix the left- and right-handed components of the quark field. It is not a symmetry of the free massless quark Lagrangian. For $z$-direction correlators the following representations of $\mathrm{SU}(2)_{C S}$ are relevant:

$$
\begin{aligned}
& R_{1}:\left\{\gamma_{1},-i \gamma_{5} \gamma_{1}, \gamma_{5}\right\} \\
& R_{2}:\left\{\gamma_{2},-i \gamma_{5} \gamma_{2}, \gamma_{5}\right\}
\end{aligned}
$$

These $R_{1}$ and $R_{2} \mathrm{SU}(2)_{C S}$ transformations connect the following operators from the $E_{2}$ multiplet:

$$
\begin{array}{ll}
R_{1}: & V_{y} \leftrightarrow T_{t} \leftrightarrow X_{t}, \\
R_{2}: & V_{x} \leftrightarrow T_{t} \leftrightarrow X_{t},
\end{array}
$$


as well as the operators from the $E_{3}$ multiplet:

$$
\begin{aligned}
& R_{1}: \quad V_{t} \leftrightarrow T_{y} \leftrightarrow X_{y}, \\
& R_{2}: \quad V_{t} \leftrightarrow T_{x} \leftrightarrow X_{x} .
\end{aligned}
$$

Our symmetry group $D_{4 h}$ includes both the permutation operator $\hat{P}_{x y}$ and $\mathbb{1}$ transformations, which form a group $S_{2}$. $\hat{P}_{x y}$ permutes $\gamma_{1}$ and $\gamma_{2}$, and transforms $\gamma_{5}$ to $-\gamma_{5}$. Then $P_{x y} R_{1}$ is isomorphic to $R_{2}$. This means that $S_{2} \times \mathrm{SU}(2)_{C S}$ contains multiplets

$$
\begin{gathered}
\left(V_{x}, V_{y}, T_{t}, X_{t}\right), \\
\left(V_{t}, T_{x}, T_{y}, X_{x}, X_{y}\right) .
\end{gathered}
$$

The degeneracy between $\mathbf{V}$ and $\mathbf{A}$ means $\mathrm{SU}(2)_{L} \times \mathrm{SU}(2)_{R}$ symmetry. A minimal group that includes $\mathrm{SU}(2)_{L} \times \mathrm{SU}(2)_{R}$ and $\mathrm{SU}(2)_{C S}$ is $\mathrm{SU}(4)$. The 15 generators of $\mathrm{SU}(4)$ are the following matrices:

$$
\left\{\left(\tau_{a} \otimes \mathbb{1}_{D}\right),\left(\mathbb{1}_{F} \otimes \Sigma_{i}\right),\left(\tau_{a} \otimes \Sigma_{i}\right)\right\}
$$

with flavour index $a=1,2,3$ and $\mathrm{SU}(2)_{C S}$ index $i=1,2,3$. Predictions of $S_{2} \times \mathrm{SU}(4)$ symmetry for isovector operators are the following multiplets:

$$
\begin{gathered}
\left(V_{x}, V_{y}, T_{t}, X_{t}, A_{x}, A_{y}\right), \\
\left(V_{t}, T_{x}, T_{y}, X_{x}, X_{y}, A_{t}\right) .
\end{gathered}
$$

$S_{2} \times \mathrm{SU}(4)$ multiplets include in addition the isoscalar partners of $V_{x}, V_{y}, T_{t}$ and $X_{t}$ operators for the first multiplet in (20) as well as of $V_{t}, T_{x}, T_{y}, X_{x}, X_{y}$ for the second multiplet in (20).

\section{Conclusions}

In this work we studied the high temperature behaviour of spatial correlation functions for connected spin $J=0$ and $J=1$ quark bilinears. Above the critial temperature we see restoration of both chiral symmetries, $\mathrm{U}(1)_{A}$ and $\mathrm{SU}(2)_{L} \times \mathrm{SU}(2)_{R}$. Additionally, some unexpected observations are made: the approximate symmetries $\mathrm{SU}(2)_{C S}$ and $\mathrm{SU}(4)$ emerge as the temperature is increased to $\sim 2 T_{c}$. At the same time there are no indications that the data would converge to the naive free quark limit.

For a discussion of implications of these observations see ref [21].

\section{Acknowledgments}

We thank C. Gattringer for numerous discussions. Support from the Austrian Science Fund (FWF) through the grants DK W1203-N16 and P26627-N27 is acknowledged. Numerical calculations are performed on Blue Gene/Q at KEK under its Large Scale Simulation Program (No. 16/17-14). This work is supported in part by JSPS KAKENHI Grant Number JP26247043 and by the Post-K supercomputer project through the Joint Institute for Computational Fundamental Science (JICFuS). G.C. is supported by STFC, grant ST/L000458/1. S.P. acknowledges the financial support from the Slovenian Research Agency ARRS (research core funding No. P1-0035). 


\section{References}

[1] G. Cossu, S. Aoki, H. Fukaya, S. Hashimoto, T. Kaneko, H. Matsufuru, J.I. Noaki, Phys. Rev. D87, 114514 (2013), [Erratum: Phys. Rev.D88,no.1,019901(2013)], 1304.6145

[2] A. Tomiya, G. Cossu, S. Aoki, H. Fukaya, S. Hashimoto, T. Kaneko, J. Noaki, Phys. Rev. D96, 034509 (2017), 1612.01908

[3] A. Bazavov et al. (HotQCD), Phys. Rev. D86, 094503 (2012), 1205. 3535

[4] T.D. Cohen, Phys. Rev. D54, R1867 (1996), hep-ph/9601216

[5] L.Ya. Glozman, Eur. Phys. J. A51, 27 (2015), 1407. 2798

[6] L.Ya. Glozman, M. Pak, Phys. Rev. D92, 016001 (2015), 1504.02323

[7] M. Denissenya, L.Ya. Glozman, C.B. Lang, Phys. Rev. D89, 077502 (2014), 1402 . 1887

[8] M. Denissenya, L.Ya. Glozman, C.B. Lang, Phys. Rev. D91, 034505 (2015), 1410 . 8751

[9] M. Denissenya, L.Ya. Glozman, M. Pak, Phys. Rev. D91, 114512 (2015), 1505.03285

[10] M. Denissenya, L.Ya. Glozman, M. Pak, Phys. Rev. D92, 074508 (2015), [Erratum: Phys. Rev.D92,no.9,099902(2015)], 1508.01413

[11] R.C. Brower, H. Neff, K. Orginos, Nucl. Phys. Proc. Suppl. 153, 191 (2006), hep-lat/0511031

[12] R.C. Brower, H. Neff, K. Orginos (2012), 1206. 5214

[13] G. Cossu, H. Fukaya, S. Hashimoto, A. Tomiya (JLQCD), Phys. Rev. D93, 034507 (2016), 1510.07395

[14] C.E. Detar, J.B. Kogut, Phys. Rev. D36, 2828 (1987)

[15] J.B. Kogut, J.F. Lagae, D.K. Sinclair, Phys. Rev. D58, 054504 (1998), hep-lat/9801020

[16] F. Karsch, E. Laermann (2003), hep-lat/0305025

[17] M. Cheng et al., Eur. Phys. J. C71, 1564 (2011), 1010. 1216

[18] K.I. Ishikawa, Y. Iwasaki, Y. Nakayama, T. Yoshie (2017), 1706.08872

[19] R.V. Gavai, S. Gupta, R. Lacaze, Phys. Rev. D78, 014502 (2008), 0803. 1368

[20] F. Karsch, E. Laermann, A. Peikert, Phys. Lett. B478, 447 (2000), hep-lat/0002003

[21] C. Rohrhofer, Y. Aoki, G. Cossu, H. Fukaya, L.Ya. Glozman, S. Hashimoto, C.B. Lang, S. Prelovsek (2017), 1707.01881 